\title{
Conference 2020: addressing a taboo subject
}

\section{Dr Ashish B. Parmar will be discussing halitosis at this year's British Dental Conference and Dentistry Show, in the Hygienist \& Therapist Symposium.}

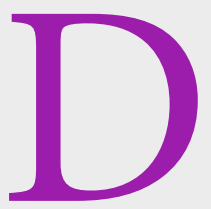

ental professionals have a choice of educational events to attend these days, so it's important to choose wisely. Be sure not to miss the British Dental Conference and Dentistry Show 2020 for all the enhanced CPD, networking and innovation you need to see you through the year.

Part of the massive appeal of this event are the exceptional speakers. The line-up for this May will be no different with a range of leading lights ready to share their insight and experience. Among them will be Dr Ashish B. Parmar. Ash is an internationally respected dentist who currently runs Smile Design By Ash in Chigwell. He is a renowned lecturer in the UK and abroad, and he has trained hundreds of dentists through The Academy By Ash. He is also a key opinion leader for various global dental companies and has featured on an array of popular TV series as a consultant and care provider.

Ash will be speaking in the Hygienist \& Therapist Symposium at the British Dental Conference and Dentistry Show 2020, sponsored by UltraDEX. His session will focus on what is a very common issue yet difficult topic to address with patients.

'I will be discussing bad breath', says Ash. 'This is a taboo subject among patients, but in around $90 \%$ of cases the cause of bad breath is related to dental problems. Therefore, as a profession, we are well-placed to help those people who are suffering.'

'What's more, helping patients overcome their issue isn't the only benefit. The concept of having fresh breath and healthy gums is more appealing than the scraping, cleaning and sometimes painful hygienist-related treatment some patients will be expecting. Instead, it is a very positive aspect for appointments and helps patients to feel good about themselves. This positive association with the dental practice and the dental hygienist and therapist can go a long way to encouraging regular hygiene maintenance visits. In turn, this enables the profession to increase awareness of dental health among the population and ultimately, to better help patients.'

During his session at the British Dental Conference and Dentistry Show, Ash will be offering practical information and advice to help the dental team deliver quality patient care.

He continues: 'Addressing bad breath can be difficult for professionals due to little training in the area and consequently a lack of knowledge about halitosis. I hope to help bridge this gap during my session. I will cover the diagnosis of bad breath, as well as management of the condition. Each member of the dental team has an important role to play. The dentist explains things to a new patient, the dental hygienist/therapist performs periodontal-related treatment thereafter, and both professionals help the patient to look after their mouth in the best way.

'I will also explore some of the products professionals can rely on to help patients effectively combat bad breath. These will include UltraDEX mouthwash. It contains chlorine dioxide to eliminate volatile sulphur compounds (VSCs) produced by plaque bacteria, which cause the bad breath.

'I hope that delegates will leave my lecture with a much better understanding of halitosis and its management. I also hope to raise awareness of the benefits of a "fresh breath" approach to patients and to demonstrate yet another reason that dental hygienists and therapists are such valuable members of the practice team.'

The Hygienist \& Therapist Symposium will present two days of lectures covering a wide range of subjects. There will be hours of enhanced CPD available both here and in the Core CPD Theatre, with additional lecture programmes catering to those with special interests in periodontology and dental implants. The Compliance Theatre will also provide useful information and guidance for anyone looking for support in this area.

Further still, the British Dental Conference and Dentistry Show 2020 will host an exhibition with more than 450 dental manufacturers and suppliers. Product experts will be on hand to demonstrate their latest innovations and help delegates maximise on investments into their technology, their business and themselves. It will also be the ideal place to network with like-minded peers who share a passion for excellence and are keen to promote best practice across all areas of dentistry.

'I would encourage dental professionals to attend this event because it presents an excellent opportunity to acquire more knowledge and information on the latest trends in dentistry', Ash adds. 'The show is excellent for all the team, including dental hygienists and therapists.'

The British Dental Conference and Dentistry Show 2020 is free for delegates to attend and will provide even more than it says on the tin. Be sure not to miss out!

Visit www.theacademybyash.co.uk to find out about the courses that Ash runs. Follow him on Instagram for his latest tips and clinical cases@ashparmardentist.

The British Dental Conference and Dentistry Show 2020 will be held on Friday 15 and Saturday 16 May at the Birmingham NEC, co-located with DTS.

For all the latest information, visit www.thedentistryshow.co.uk, call 02073485270 or email dentistry@ closerstillmedia.com.

https://doi.org/10.1038/s41407-020-0261-9 